\title{
Ground calibration of the spatial response and quantum efficiency of the CdZnTe hard x-ray detectors for NuSTAR
}

Brian W. Grefenstette, Varun Bhalerao, W. Rick Cook, Fiona A. Harrison, Takao Kitaguchi, et al.

Brian W. Grefenstette, Varun Bhalerao, W. Rick Cook, Fiona A. Harrison, Takao Kitaguchi, Kristin K. Madsen, Peter H. Mao, Hiromasa Miyasaka, Vikram Rana, "Ground calibration of the spatial response and quantum efficiency of the CdZnTe hard x-ray detectors for NuSTAR," Proc. SPIE 10392, Hard X-Ray, Gamma-Ray, and Neutron Detector Physics XIX, 1039207 (29 August 2017); doi: 10.1117/12.2271365

SPIE Event: SPIE Optical Engineering + Applications, 2017, San Diego, California, United States 


\title{
Ground Calibration of the Spatial Response and Quantum Efficiency of the CdZnTe Hard X-ray Detectors for NuSTAR
}

\author{
Brian W. Grefenstette ${ }^{1}$, Varun Bhalerao ${ }^{2}$, W. Rick Cook $^{1}$, Fiona A. Harrison ${ }^{1}$, Takao \\ Kitaguchi ${ }^{3}$, Kristin K. Madsen ${ }^{1}$, Peter H. Mao ${ }^{1}$, Hiromasa Miyasaka ${ }^{1}$, Vikram Rana ${ }^{1}$ \\ ${ }^{1}$ Space Radiation Lab, California Institute of Technology (Caltech), Pasadena, CA \\ ${ }^{2}$ Department of Physics, Indian Institute of Technology Bombay, Powai, Mumbai, India \\ ${ }^{3}$ RIKEN Nishina Center, 2-1 Hirosawa, Wako, Saitama 351-0198, Japan
}

\begin{abstract}
Pixelated Cadmium Zinc Telluride (CdZnTe) detectors are currently flying on the Nuclear Spectroscopic Telescope ARray $(N u S T A R)$ NASA Astrophysics Small Explorer. While the pixel pitch of the detectors is $\approx 605$ $\mu \mathrm{m}$, we can leverage the detector readout architecture to determine the interaction location of an individual photon to much higher spatial accuracy. The sub-pixel spatial location allows us to finely oversample the point spread function of the optics and reduces imaging artifacts due to pixelation. In this paper we demonstrate how the sub-pixel information is obtained, how the detectors were calibrated, and provide ground verification of the quantum efficiency of our Monte Carlo model of the detector response.
\end{abstract}

\section{INTRODUCTION}

The advent of imaging hard X-ray telescopes has brought a revolution in the sensitive study of the X-ray sky at energies beyond $10 \mathrm{keV}$. The focusing optics flown on the Nuclear Spectroscopic Telescope ARray (NuSTAR) NASA Astrophysics Small Explorer ${ }^{1}$ produce a point spread function (PSF) with a Gaussian-like core with a $1 \sigma$ width of $\approx 7^{\prime \prime}$ and broad wings that result in a half-power diameter of $\approx 60^{\prime \prime}{ }^{2}$. Taking full advantage of the narrow Gaussian core requires focal plane detectors that provide position information on comparable fine spatial scales. For the $10-\mathrm{m}$ NuSTAR focal length, the $0.6048 \mathrm{~mm}$ pitch of the $N u S T A R$ pixels ${ }^{3}$ corresponds to an angular size of $\approx 12^{\prime \prime}$. Even with the natural dither provided by the motions of the NuSTAR mast, this is far too coarse. We can leverage the properties of the detectors to improve our knowledge of the interaction location.

Unlike in other solid state detectors (such as Si-based CCDs), the location and size of a pixel of our Cadmium Zinc Telluride (CZT) detectors are not defined by physical boundaries (such as etched streets in a Si wafer). The CZT is, instead, a monolithic crystal where pixelation is achieved by segmenting the anode readout and then applying a electric field across the crystal. When a photon interacts in the active volume it produces a charge cloud that drifts along the internal electric fields lines to the anode, where the charge is collected. The location and extent of any individual pixel can only, therefore, be determined by illuminating the crystal with a highly collimated X-ray source with a known position to determine the regions of the crystal that correspond to a given readout pixel.

For the NuSTAR detectors, the CZT crystals are read out by a custom, low-noise application specific integrated circuit (ASIC) ${ }^{4,5}$ (hereafter we refer to the CZT/ASIC combination as a "hybrid") that determines the pixel where the most charge was collected and reads out that pixel along with its eight nearest neighbors. For photons that arrive in the center of the pixel, all of the charge is collected by the central pixel and so we can determine the centroid of the region that corresponds to this pixel. However, for pixels where some of the charge is collected by both the central pixel and one (or more) of its nearest neighbors (a "split pixel" event), we know that the photon must have interacted near the boundary between the pixels. We can leverage this to achieve sub-pixel spatial resolution on length scales necessary to properly oversample the NUSTAR PSF.

In this paper we describe the laboratory setup used to calibrate the NuSTAR detectors, the architecture used to determine the sub-pixel information, and how the final calibration was performed.

Hard X-Ray, Gamma-Ray, and Neutron Detector Physics XIX, edited by Arnold Burger, Ralph B. James, Michael Fiederle, Larry Franks, Stephen A. Payne, Proc. of SPIE Vol. 10392, 1039207

(c) 2017 SPIE · CCC code: 0277-786X/17/\$18 - doi: 10.1117/12.2271365 


\section{XRG SCANS}

\subsection{Experimental Setup}

We use a pencil beam scan to determine the spatial response of the detectors. The X-ray beam is produced using a Rigaku X-ray generator with a Mo tube. The beam was operated at its lowest settings $(20 \mathrm{kV}$ bias and $2 \mathrm{~mA}$ current) to avoid saturating the CZT detectors. This results in a featureless continuum spectrum with a maximum energy of $20 \mathrm{keV}$. From the X-ray generator tower the beam is directed through a $39 \mathrm{~cm}$ beam pipe at the end of which the beam hits a pair of Tungsten slits that collimate the beam down to spot.

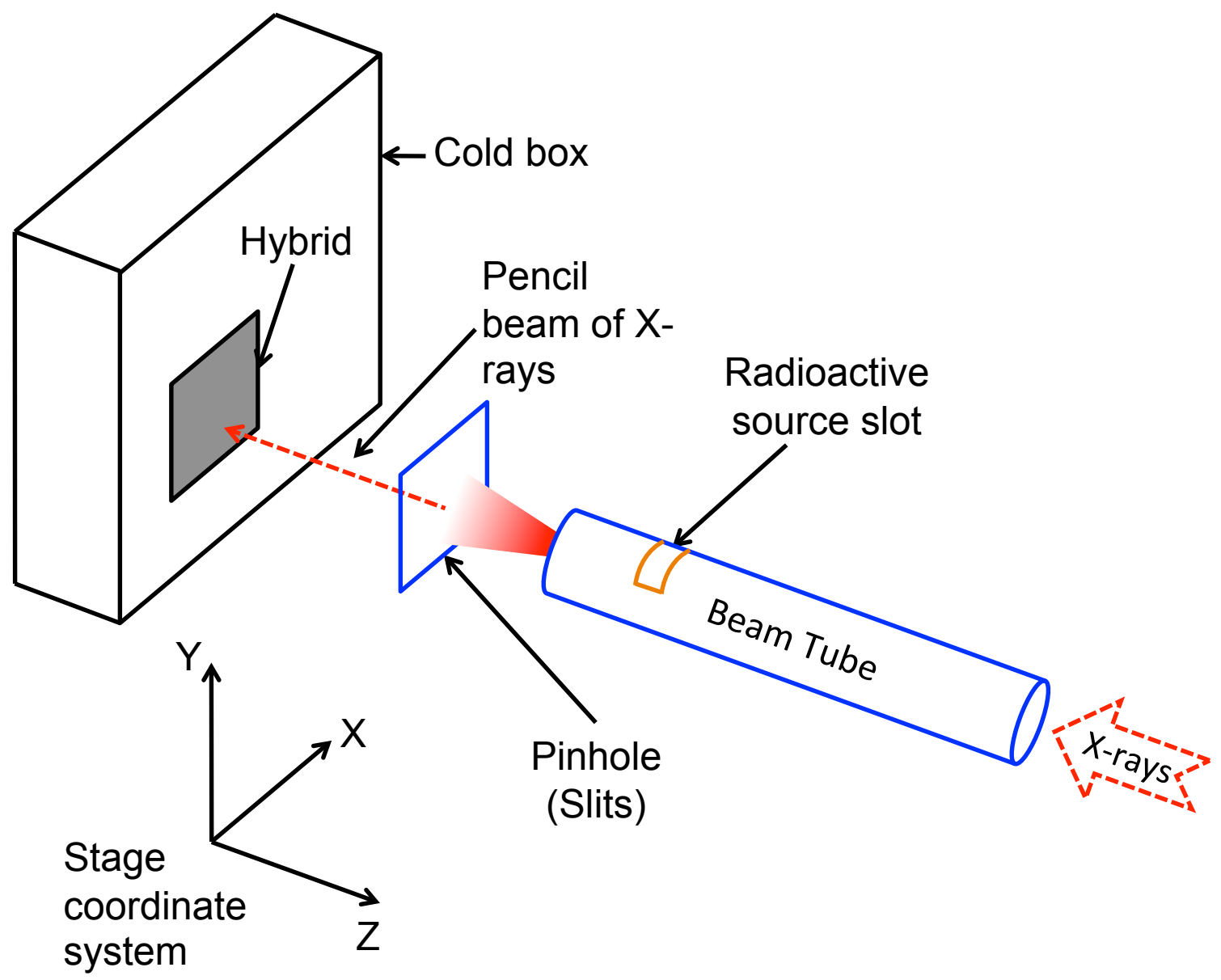

Figure 1. A schematic of the beam setup. X-rays enter from the X-ray generator on the lower right and pass through the hollow beam tube. Once passing through the slits the pencil beam shines on the CZT Hybrid detector. The cold box is mounted on $\mathrm{X} / \mathrm{Y}$ translation stages which move the cold box and move the beam across the hybrid. A slot in the beam pipe allowed us to illuminate the hybrid with a known radioactive source for the quantum efficiency calibration. This figure has been reproduced from ${ }^{6}$.

To determine the profile of the beam as it hits the detector we perform a 1-D knife edge scan. Rather than scan across the CZT detector, we instead place a Amptek XR-100 CR Si-PIN detector so that all of the beam photons hit the $2 \times 2 \mathrm{~mm}$ Si detector. We then use another pair of translation stages to step the knife-edge across the Si detector, integrating for long enough at each position so that statistical errors are small. The resulting 1-D scan then has the functional form of an integrated 1-D Gaussian. In IDL this can be fit using the erf function which is parameterized by the peak of the Gaussian distribution (i.e. the mean value of the Gaussian) and the 
1- $\sigma$ width of the Gaussian. The scan is repeated in both the X-and Y-directions, which results in fits of 50 and $80 \mu \mathrm{m}$, respectively.
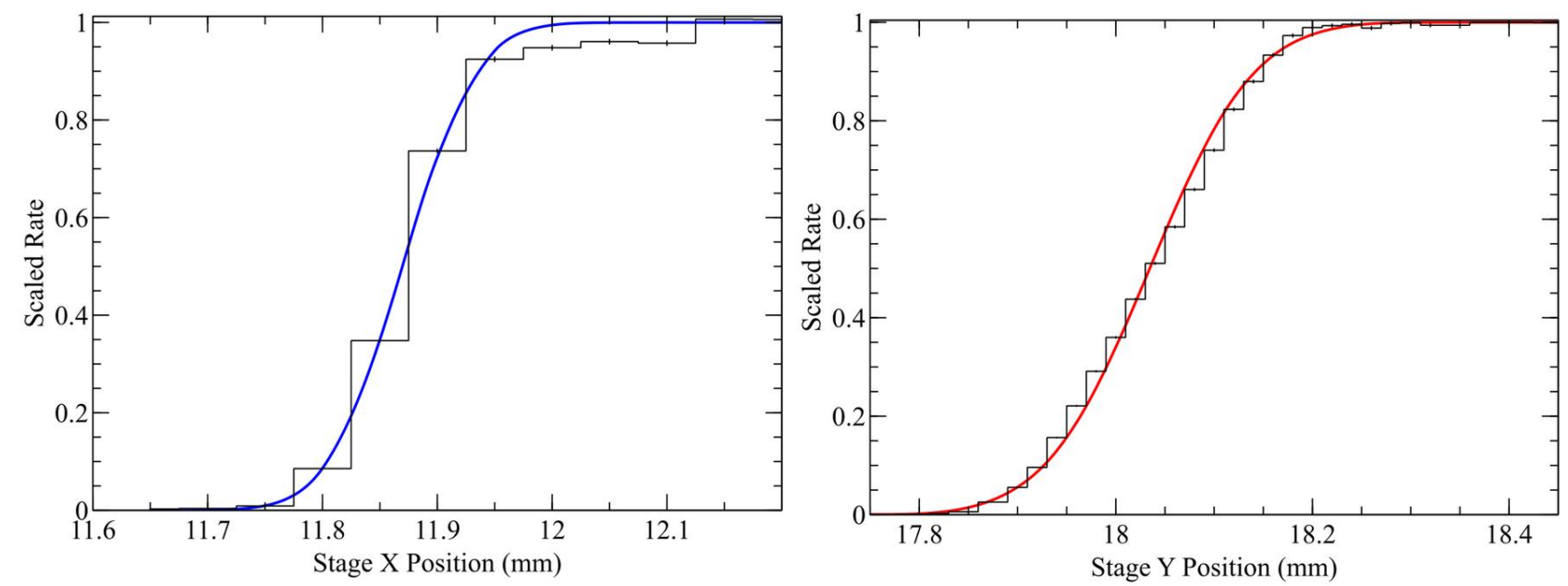

Figure 2. The beam profile for the X-and Y-direction scans of the blocking filter across the beam. The best-fit integrated Gaussian profiles (see the text) are shown in blue and red for the the X-and Y-direction scans of the beam along with $1-\sigma$ error bars.

To scan the beam across the detectors we mount a CZT detector inside of an cold box that maintains the detectors at their operating conditions (nominally $3-5{ }^{\circ} \mathrm{C}$ ). The entire setup is located in an enclosure that is purged with $\mathrm{N}_{2}$ gas and kept at positive pressure to prevent water condensation on the detectors or the readout electronics.

The detectors are read out using a laboratory setup that mimics the behavior of the flight electronics and stores the data in a nearly identical data format to that of the data obtained in flight. The entire enclosure is then mounted on a pair of Newport LTA-HS linear translation stages that have an absolute accuracy of $\pm 7.5 \mu \mathrm{m}$ and repeatability of $\pm 1 \mu \mathrm{m}$. The total traverse of each stage is $50 \mathrm{~mm}$, which is sufficient to scan the $\approx 20 \mathrm{~mm}$ active detector area. The stages are driven by a Newport ESP-300 controller which is commanded by custom $\mathrm{C \#}$ software on a laboratory PC.

We scan over each detector in both the $\mathrm{X}$ - and Y-directions in a raster pattern with the scan pattern extending beyond the nominal detector size to ensure that we fully scan the active area of each detector. While scanning in the $X$ direction, the beam was moved at $150 \mu \mathrm{m} s^{-1}$, then stepped up by $75 \mu \mathrm{m}$ (roughly one beam width) for the next scan. For vertical scans, the beam moved at $225 \mu \mathrm{m} \mathrm{s}{ }^{-1}$, then moved sideways by one beam width $50 \mu \mathrm{m}$ for the next pass (See Figure 3).

The X-ray events and the position of the $\mathrm{X} / \mathrm{Y}$ stages are both time tagged by the data acquisition electronics so we can interpolate the stage locations onto the photon arrival time in post-processing. The raw event data are processed using code similar to the NuSTARDAS flight software to produce a list of events where the row and column of the central pixel of the $3 \times 3$ readout is stored, as is the energy of the event, the STAGE positions at the time the photon arrived, and the "grade" of the event. Here the grade is used to identify the specific morphology of events where charge is shared between multiple pixels (Figure 4, left panel). This grade distribution can then be used to identify sub-regions of the pixel that will produce a particular grade morphology (Figure 4, right panel).

\subsection{Pixel Location Determination}

The purpose of this analysis is to determine the interaction location for a count that was registered by a given detector, in a given pixel, and with a particular grade. To accomplish this, we first define the grid that will 


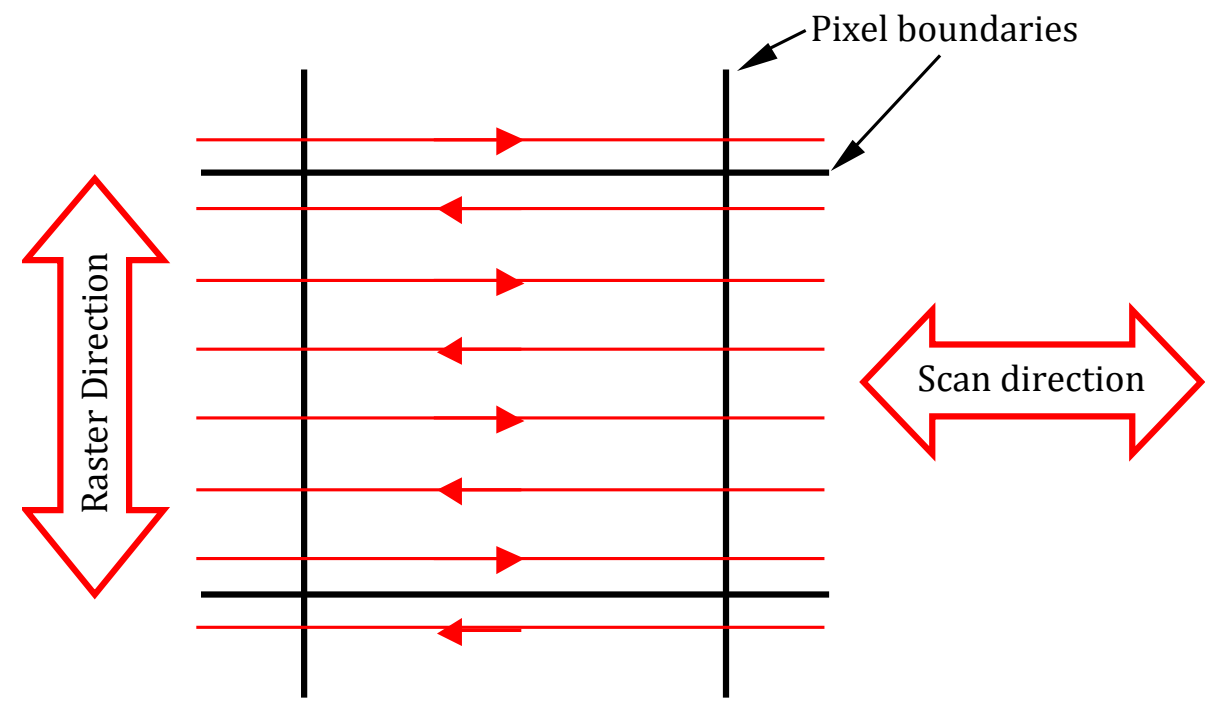

Figure 3. The black lines represent the fiducial boundary of the pixel, while the red lines show the path of the X-ray beam across the detector. After each pass, the beam is moved in the raster direction and another scan across the detector is performed. Once the detector has been completely scanned in the horizontal direction the process is repeated with the scan and raster axes switched. Figure reproduced from ${ }^{6}$.

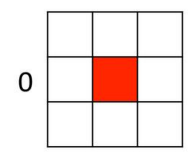

1

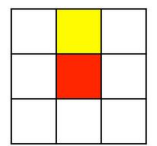

2

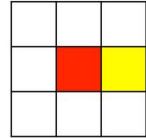

3
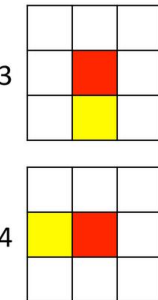

5

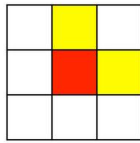

6

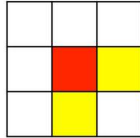

7

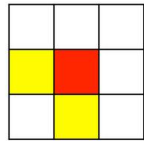

8

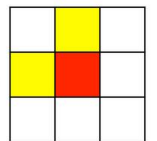

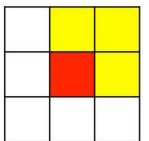

10

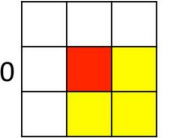

11

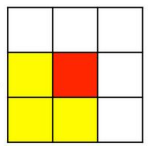

12

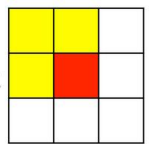

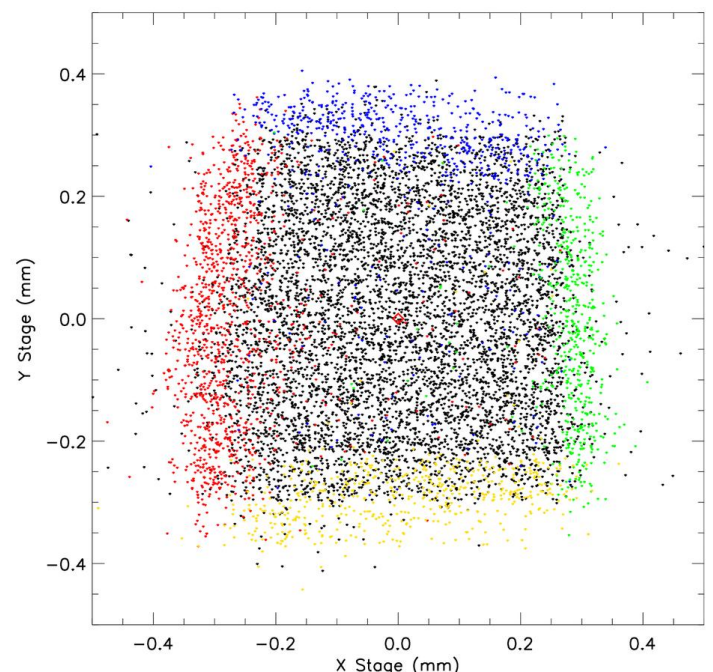

Figure 4. (Left): The definition of the "science" grades used for NuSTAR data analysis. The red squares represent the pixel that collects the most charge from the photon interaction, the yellow squares represent pixels that are above the software trigger threshold for collecting charge, while the white squares are below the trigger threshold. (Right) The data from both scan directions are shown for a single pixel with grades $0,1,2,3$, and 4 drawn as black, blue, green, yellow, and red dots. The red diamond shows the center of the pixel's effective area, while the data points are shown in the STAGE coordinates relative to the center of the pixel. The data points locations have been blurred with uniform random distribution with widths of 50 and $70 \mu \mathrm{m}$ in the $\mathrm{X}$ - and $\mathrm{Y}$ - directions, respectively.

define the focal plane coordinate system. Based on early scan data, we determined that oversampling a physical pixel by a factor of five would provide sufficient spatial resolution to properly over sample the optics PSF.

Each CZT detector contains a grid of $32 \times 32$ pixels, while a full NuSTAR focal plane is composed of a four detectors mounted into a 2x2 array (Figure 5). We define the DET1 coordinate system to be a 360x360 grid centered on the physical focal plane with a grid spacing of $0.12096 \mathrm{~mm}$ (one fifth of the nominal physical pixel 
size). This grid size allows us to cover the entire active area on the focal plane.

The goal is to determine a probability density function (PDF) in the DET1 coordinate grid for each pixelgrade combination. We assign a position for each count by applying a uniform random offset to the linear stage coordinates with length scales of 50 and $70 \mu \mathrm{m}$ in the X- and Y-directions, respectively, to account for the width of the XRG beam. This is slightly flatter than the Gaussian profile measured above, which we take to be a conservative estimate of our knowledge of the stage locations at the time the count was detected.

This resulting event location is in a STAGE coordinate system that can be related to the DET1 coordinate system through a simple rotation and subsequent translation. After these operations are complete, we then bin the resulting data into the DET1 coordinate system. We find that we can capture nearly all of the counts in a $7 \times 7$ subgrid of the DET1 coordinate system, which we can divide by the total number of observed counts to produce the PDF for that pixel and grade. The PDF is then stored in a calibration data file for later use (hereafter, the PIXPOS file).
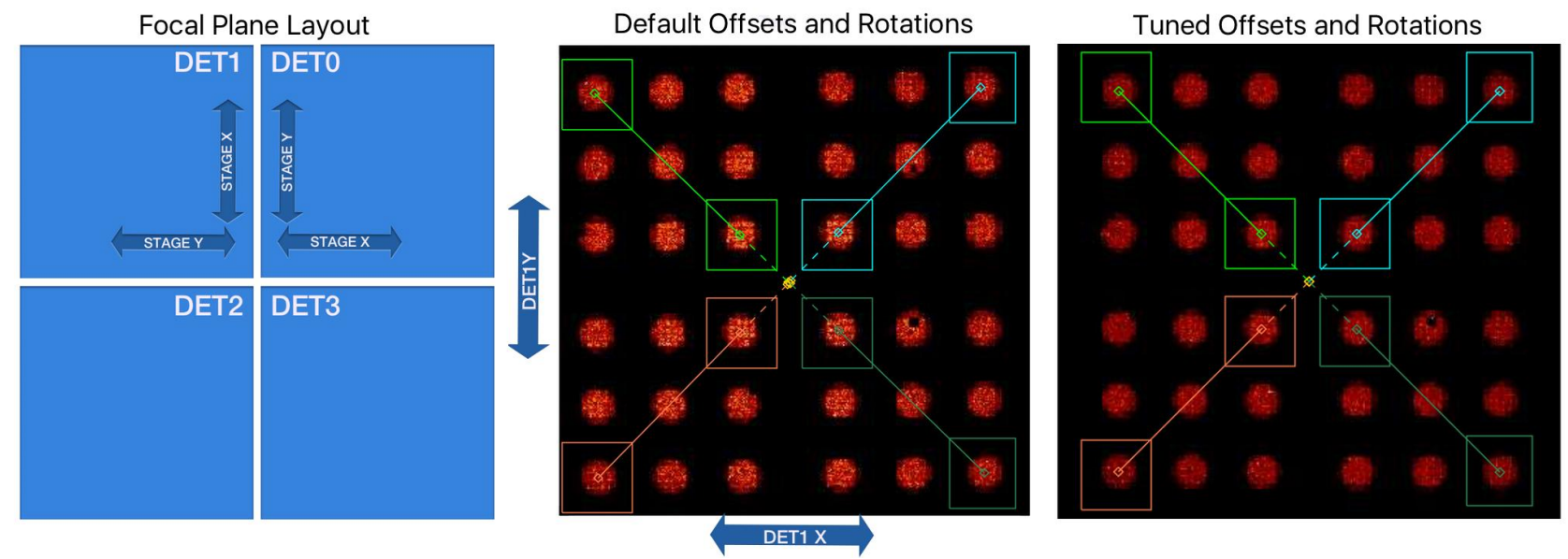

Figure 5. (Left) The layout of a NuSTAR focal plane showing the the nominal location rotation and offset between all four focal plane detectors. (Middle) The layout location of the projected spots from the alignment plate assuming nominal offsets and rotations, the regions used to determine the centroid of the alignment spots (boxes), and the estimated location of the origin based on the spots on each detector (gold diamonds). There is also an overall rotation of $\approx 0.5$ degrees that represents the absolute rotation between the stage coordinate systems and the DET1 coordinates. (Right) The layout of the projected spots after all the corrections have been made to the STAGE coordinates and the PDFs have been recomputed in the DET1 coordinate system. Data shown are shown for one of the two focal plane modules, but are representative of both focal planes.

\subsection{Absolute Alignment}

After all four detectors are mounted in the flight focal plane, we use a radioactive ${ }^{55} \mathrm{Fe}$ source placed above a uniformly machined aluminum alignment plate to determine the absolute position of each detector. The plate consists of four sets of $3 \times 3$ grids of holes that have center-to-center separations of $6 \mathrm{~mm}$. The radioactive source is placed $300 \mathrm{~mm}$ (roughly 1-ft) away from the center of the alignment plate. This results in spots being projected on the surface of the detectors with a known geometry.

The nominal configuration (left panel in Figure 5) is that the detectors are rotated by $90^{\circ}$ with respect to their neighbors with a "street" of roughly $100 \mu \mathrm{m}$ separating a detector from its neighbors. Using these assumptions we can project the locations of the alignment data into DET1 coordinates (middle panel of Figure 5).

We measure the mean position of each projected spot in both the DET1X and DET1Y directions for two spots for each focal plane. The spots contain enough counts $(>15,000)$ so that any uncertainties from the digitization due to the $120.95 \mu \mathrm{m}$ grid spacing are on the order of $1 \mu \mathrm{m}$.

Using the centroids of two spot for each detector we can easily determine any residual rotation and offset with respect to the DET1 coordinate system. We re-generate the PIXPOS file after applying rotations and then 
translations to the data for each of the four detectors. After applying these corrections to the STAGE coordinates for each detector we can generate a new PIXPOS file, re-project the alignment data and finally determine the absolute offset of each detector's STAGE coordinates with respect to the DET1 coordinates. After the final iteration has been applied to the data the four detectors agree on the location of the center of the focal plane to an accuracy roughly $5 \mu \mathrm{m}$ (or roughly $0.2 \operatorname{arcsec}$ ).

\section{QUANTUM EFFICIENCY}

The instrument response for the NuSTAR detectors is based on a model of the detectors that combines a Monte Carlo simulation of the X-ray interactions with the subsequent charge transport within the pixel ${ }^{7}$. Here we wish to verify that the absolute calibration of the detectors are consistent with the Monte Carlo model of the instrument response.

\subsection{Experimental Setup}

The absolute quantum efficiency is verified using a reference ${ }^{241} \mathrm{Am}$ radioactive source illuminating a $N u S T A R$ hybrid through a set of tungsten slits with an opening of $5 \mathrm{~mm}(0.2$ ") (Figure 6, hereafter the "QE Mask"). This setup produces a square illuminated region on the surface of the CZT detectors. The ${ }^{241} \mathrm{Am}$ source is composed of a dot of radioactive material suspended in a resin holder, which results in line emission associated with the ${ }^{241} \mathrm{Am}$ decay at $59.54 \mathrm{keV}$ as well as a down-scattered continuum from the primary gamma-rays scattering in the resin and the holder (Figure 7, left).
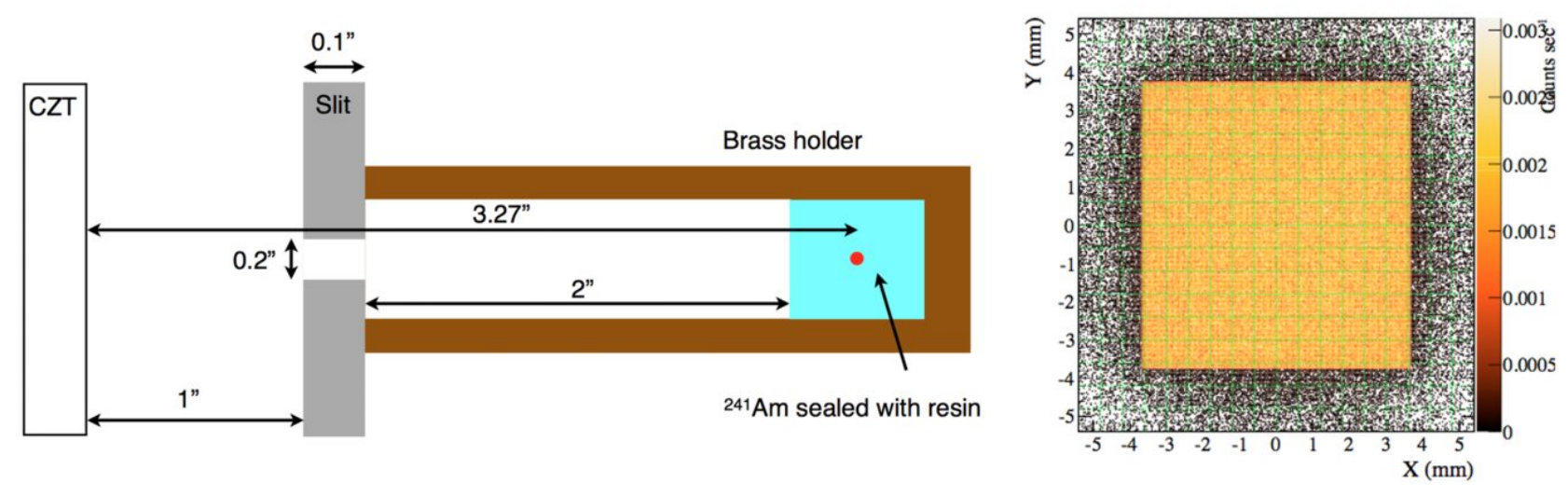

Figure 6. (Left) A schematic view of the ${ }^{241} \mathrm{Am}$ X-ray source (red dot), the resin that surrounds the source (cyan), the brass source holder, and the tungsten slits (grey) with the dimensions given in inches ("). (Right) A simulation showing an intensity map of the ${ }^{241} \mathrm{Am}$ source counts on the detector plane (CZT or Ge) along with a uniform grid (green) showing the nominal boundaries between pixels.

\subsection{Setup Calibration}

We construct a GEANT $4{ }^{8}$ model of the QE mask. This includes the source emission lines ${ }^{9}$ and a mass model of the enclosing resin, the brass holder, and the tungsten slits, along with a GEANT4 model of a reference Ortec GMX series Gamma-X HPGe detector. The relatively simple response of the Ge detector and its associated electronics allows us to determine the shape and the absolute flux of the radioactive source at $59.54 \mathrm{keV}$. In the region around the primary $59.54 \mathrm{keV}$ line (Figure 7, right) we find that the source is producing $48.76 \pm 0.28 \mathrm{ph}$ $\mathrm{sec}^{-1}$ incident on the Ge detector through the QE mask.

\subsection{NuSTAR Hybrid Analysis}

The QE mask replaces the pin hole slit used in the XRG analysis above. As with the pencil beam, we translate each hybrid so that the QE mask illuminates different regions of the CZT detector. This allows us to select a 

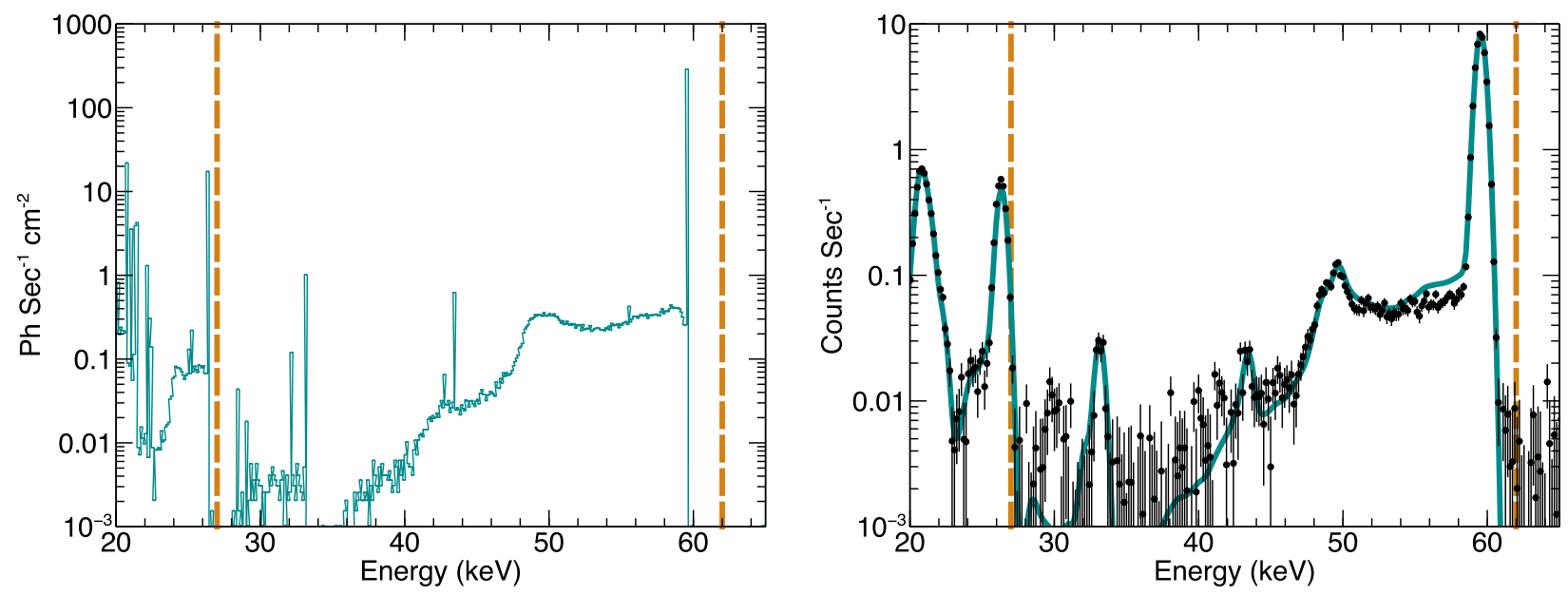

Figure 7. (Left) Simulated ${ }^{241}$ Am photon spectrum at the detector plane. The vertical dashed lines show the $27-62$ $\mathrm{keV}$ bandpass used to analyze the data. (Right) The data from the Ge detector (black with 1- $\sigma$ errors) along with the simulated spectrum convolved with the Ge detector response and a Gaussian kernel to model the noise in the readout electronics.

region near the center of the CZT detector that is also free of disabled pixels so that we can safely assume that all photons that pass through the QE mask will be detected by the CZT.

We integrate over the 27-62 keV bandpass to cover the escape peaks from the CZT detectors from a $60 \mathrm{keV}$ monochromatic spectrum (Figure 8). We then compare the observed rate to the expected rate based on the Ge calibration to determine the QE of each hybrid.

Errors in the count rate are assumed to be Gaussian (reasonable as the number of observed counts is typically $\gg$ 10000). Table 1 shows the results for the "best" conditions where the source image is projected in the interior of the detector, a reasonable number (typically 1 or 0 ) of pixels are disabled, and we can directly compare the Ge and CZT results. The variation in the number of illuminated pixels arises from some small variations in the distance between the CZT and the QE mask as the detectors are mounted in the calibration setup.

Also given in Table 1 is the expected QE from the detector response matrix files (RMFs) produced by our Monte Carlo simulation of the detector response ${ }^{7}$ that has been tuned for each detector based on the detector thickness and the charge transport properties.

With the exception of FPMB2 all of the detectors measure an incident rate that is consistent with our expectations. For FPMB2 there were both noisy pixels that were persistently triggering throughout the ground calibration observation as well as a large number of disabled pixels during the QE observation even in the best case. We believe this is artificially reducing the quantum efficiency of the detector.

\section{SUMMARY}

We performed calibration measurements of all 8 NuSTAR focal plane hybrids during a ground calibration campaign in 2010 and 2011 prior to launch. Our results are:

1. We leverage the fact that the $N u S T A R$ readout electronics identify events where charge is share between neighboring pixels in the CdZnTe detectors to achieve sub-pixel localization of individual X-ray photons.

2. We combine the X-ray generator scan of each individual detector and the illumination of the fully populated focal plane array to measure the relative alignment of the detectors to better than $10 \mu \mathrm{m}$.

3. For events without charge sharing (black data points in in Figure 4), events can be localized to the interior $\approx 400 \mu \mathrm{m}$ of each pixel. 


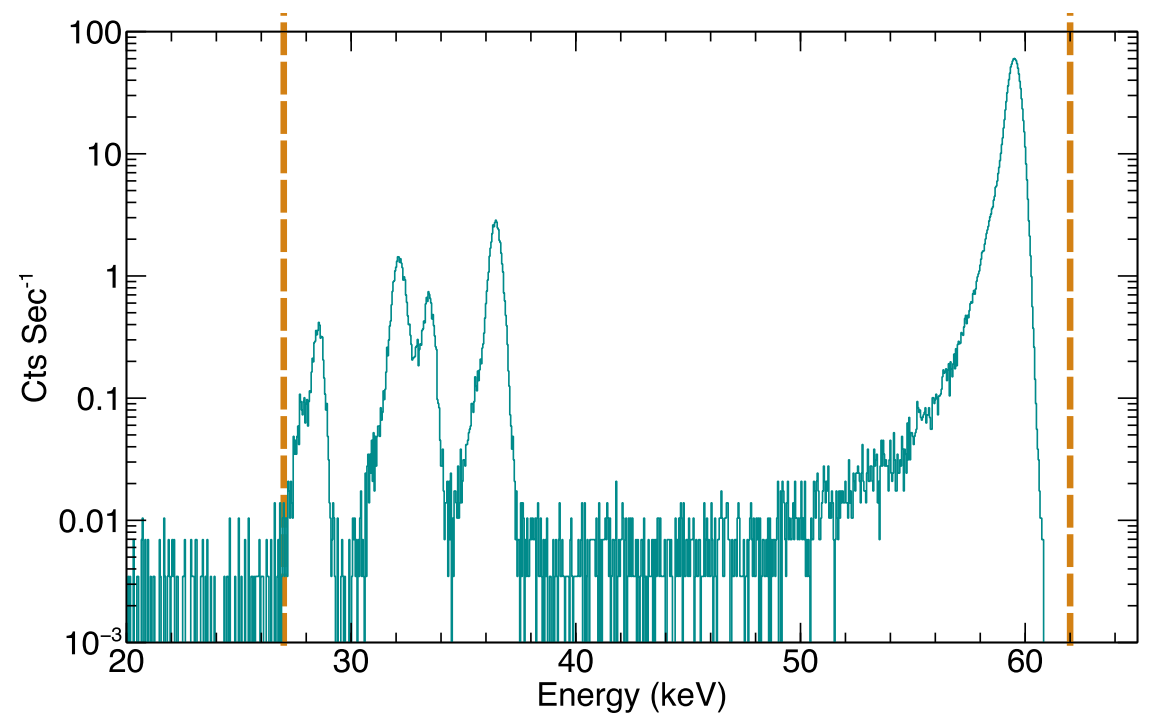

Figure 8. In green we show the simulated spectrum observed by a NuSTAR CZT detector to a monochromatic $60 \mathrm{keV}$ line. The spectrum has been convolved with the $C Z T$ response, which includes the presence of the secondary peaks from 28-36 keV that correspond to the escape lines from $\mathrm{Cd}$ and Te from $23-31 \mathrm{keV}$ below the $60 \mathrm{keV}$ photopeak. The vertical dashed lines show the energy range used for the CZT analysis and are the same as in Figure 7.

Table 1. Quantum Efficiency at $60 \mathrm{keV}$

\begin{tabular}{|c|c|c|c|c|c|c|}
\hline Detector & Epoch & Active Pixels $^{a}$ & Observed Rate & Incident Rate $^{b}$ & Measured QE (\%) & Expected QE (\%) \\
\hline $\mathrm{Ge}$ & $2012-01-27$ & & $47.81 \pm 0.11$ & $48.76 \pm 0.27$ & & \\
\hline FPMA0 & 2011-02-28 & 265 & $48.74 \pm 0.19$ & $48.86 \pm 0.28$ & $99.7 \pm 0.7$ & 99.6 \\
\hline FPMA1 & 2011-09-21 & 281 & $48.68 \pm 0.16$ & $48.80 \pm 0.28$ & $99.8 \pm 0.7$ & 99.2 \\
\hline FPMA2 & 2011-08-24 & 252 & $49.00 \pm 0.23$ & $"$ & $100.6 \pm 0.8$ & 99.5 \\
\hline FPMA3 & 2011-09-14 & 284 & $48.45 \pm 0.16$ & $"$ & $99.3 \pm 0.7$ & 99.3 \\
\hline FPMB0 & 2011-01-24 & 260 & $48.65 \pm 0.17$ & $48.87 \pm 0.28$ & $99.5 \pm 0.7$ & 99.5 \\
\hline FPMB1 & 2011-01-29 & 254 & $48.58 \pm 0.19$ & $"$ & $99.4 \pm 0.7$ & 99.7 \\
\hline FPMB2 & 2011-01-21 & 272 & $47.05 \pm 0.16$ & $"$ & $96.3 \pm 0.6$ & 99.7 \\
\hline FPMB3 & 2011-01-31 & 272 & $48.47 \pm 0.19$ & $"$ & $99.2 \pm 0.7$ & 99.6 \\
\hline
\end{tabular}

$a$ The number of "active" pixels is defined to be the number of pixels that recorded at least 100 counts.

$b$ : For Ge detector the incident rate is the incident photon flux as inferred from the GEANT4 mass model. For the CZT detectors the Ge photon flux has been scaled to account for the change in the activity of the ${ }^{241}$ Am source between the epoch of the Ge measurement and the epochs of the CZT measurements.

4. Events with charge shared between multiple pixels (colors in Figure 4) come from smaller physical regions of each pixel, and so we can better localize these events.

5. All of the NUSTAR hybrids have a measured quantum efficiency with respect to a reference Ge detector of near unity at $60 \mathrm{keV}$ and agree with our models of the detector response except for a single detector that exhibited poor performance in the calibration setup.

\section{ACKNOWLEDGEMENTS}

This work was supported under NASA Contract No. NNG08FD60C, and made use of data from the NuSTAR mission, a project led by the California Institute of Technology, managed by the Jet Propulsion Laboratory, and funded by the National Aeronautics and Space Administration. 


\section{References}

1.F. A. Harrison et al., "THE NUCLEAR SPECTROSCOPIC TELESCOPE ARRAY ( NuSTAR ) HIGHENERGY X-RAY MISSION", The Astrophysical Journal 770(2), 103, IOP Publishing (2013) [doi:10.1088/0004$637 \mathrm{x} / 770 / 2 / 103]$.

2.K. K. Madsen et al., "CALIBRATION OF THE NuSTAR HIGH-ENERGY FOCUSING X-RAY TELESCOPE", The Astrophysical Journal Supplement Series 220(1), 8, IOP Publishing (2015) [doi:10.1088/00670049/220/1/8].

3.V. R. Rana et al., "Development of focal plane detectors for the Nuclear Spectroscopic Telescope Array (NuSTAR) mission", in UV X-Ray and Gamma-Ray Space Instrumentation for Astronomy XVI, O. H. Siegmund, Ed., SPIE-Intl Soc Optical Eng (2009) [doi:10.1117/12.825418].

4.W. R. Cook et al., "High resolution CdZnTe pixel detectors with VLSI readout", IEEE Transactions on Nuclear Science 47(4), 1454-1457, Institute of Electrical and Electronics Engineers (IEEE) (2000) [doi:10.1109/23.872995].

5.H. Miyasaka et al., "Development of a cadmium telluride pixel detector for astrophysical applications", in UV X-Ray and Gamma-Ray Space Instrumentation for Astronomy XVI, O. H. Siegmund, Ed., SPIE-Intl Soc Optical Eng (2009) [doi:10.1117/12.825711].

6.V. Bhalerao, "Neutron Stars and NuSTAR", PhD thesis, Caltech (2012).

7.T. Kitaguchi et al., "Spectral calibration and modeling of the NuSTAR CdZnTe pixel detectors", in UV X-Ray and Gamma-Ray Space Instrumentation for Astronomy XVII, O. H. Siegmund, Ed., SPIE (2011) [doi:10.1117/12.896972].

8.S. Agostinelli et al., "Geant4 - a simulation toolkit", Nuclear Instruments and Methods in Physics Research Section A: Accelerators Spectrometers, Detectors and Associated Equipment 506(3), 250-303, Elsevier BV (2003) [doi:10.1016/s0168-9002(03)01368-8].

9.M. C. Lépy, J. Plagnard, and L. Ferreux, "Measurement of 241Am L X-ray emission probabilities", Applied Radiation and Isotopes 66(6-7), 715-721, Elsevier BV (2008) [doi:10.1016/j.apradiso.2008.02.078]. 